\title{
RARE DECAYS OF THE Z AT LEP
}

\author{
Tofigh Azemoon \\ Department of Physics \\ University of Michigan \\ Ann Arbor, MI 48109, USA
}

\begin{abstract}
I discuss the preliminary results of the LEP experiments ALEPH, DELPHI, L3 and OPAL on the rare $Z$ decays. Limits of about $10^{-5}$ on the branching ratios are reported by most experiments. The question of excess in the $\tau \tau \mathrm{V}$ final state remains unsettled. From the analysis of the L3 data the $\mathrm{ZZ}^{\prime}$ mixing angle is confined to $\pm 1.5^{\circ}$ for most extensions of the Standard Model of electroweak interactions.
\end{abstract}

\section{INTRODUCTION}

In this note we consider the $\mathrm{Z}$ decays that are either forbidden or have very small partial widths in the current theories such that a positive signal in the present LEP data would be interpreted as the manifestation of new physics. We also review the search for anomalies in the four fermion final states near the $\mathrm{Z}$ pole and present the limits on $\mathrm{Z}^{\prime}$ parameters from direct line-shape measurements by the L3 collaboration.

\section{LEPTON FLAVOUR VIOLATING DECAYS}

So far, all experimental results support the concept that the $e, \mu$ and $\tau$ lepton numbers are individually conserved. At the same time the extensions to the Standard Model, such as ones with exotic gauge bosons or fermions, predict branching ratios as large as $10^{-4}$ for the lepton flavour violating decays: $\mathrm{Z} \rightarrow \mathrm{e} \mu, \mathrm{e} \tau$ and $\mu \tau .{ }^{1}$ Experimentally, the largest source of background for the $e \tau$ and $\mu \tau$ decays is the $\mathrm{Z} \rightarrow \tau \tau$ channel where one $\tau$ decays into $e \nu \nu$ or $\mu \nu \nu$ with the electron or muon energy near the allowed maximum. Hence a good electron and muon energy resolution is crucial for the above measurements. This is demonstrated in figure 1 from the L3 experiment showing the electron energy in $\mathrm{Z} \rightarrow e \tau$ and from the $\tau$ decay in $Z \rightarrow \tau \tau$. Although for clarity only the last few bins of the background spectra are shown, the normalization is over the entire range. The energy resolution is $1 \%$ for $45 \mathrm{GeV}$ electrons and with a $2 \sigma$ cut on the electron energy only one candidate is accepted in the data, where 1.2 events are expected from the $\tau \tau$ Monte Carlo. The results of the four experiments ${ }^{2-5}$ are given in table 1. For comparison we also show the limits on the $\mathrm{Z}$ branching ratios estimated from the measurement of the low energy processes listed in the table. These are the updated values of the limits given in ref. 6 using the most recent entries in ref. 7. For $\mathrm{Z} \rightarrow e \tau$ and $\mu \tau$ the LEP limits are more stringent than those extracted from the low energy data. For $Z \rightarrow e \mu$ the LEP sensitivity cannot match that of the low energy measurements. However, it is worth noting 


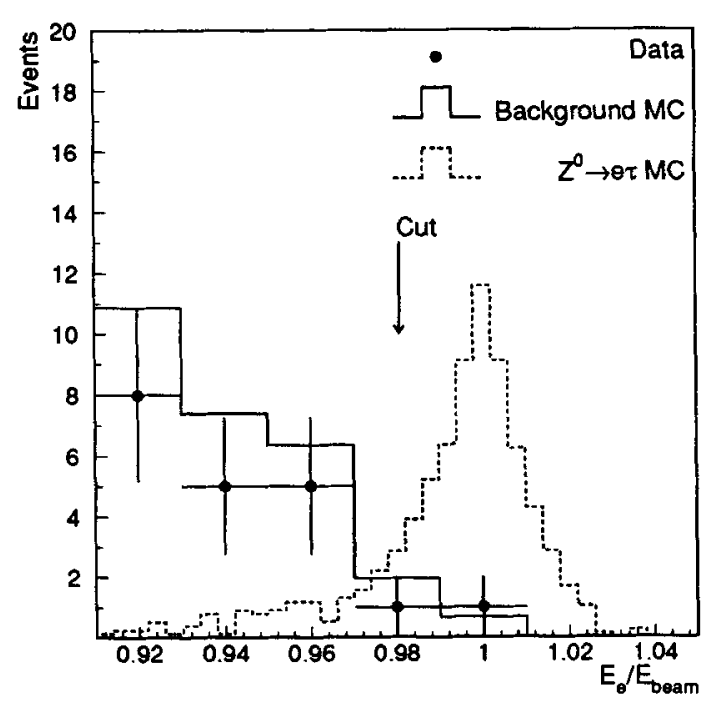

Figure 1. Electron energy spectrum from L3 experiment. Solid hystogram is the $\tau \tau$ Monte Carlo and the solid points are the $\tau \tau$ data.

that the measurements involving light particle decays are not sensitive to all lepton flavour violating couplings as some of those appear with small coefficients in the Lagrangian. ${ }^{6}$ For the $\mathrm{Z}$ decays all coefficients are comparable, and if the couplings that are unconstrained by the low energy data happen to be anomalously large the extracted limits would be invalid. Hence it is important to measure all three branching ratios directly at LEP.

\section{Z DECAYS WITH A VECTOR BOSON IN THE FINAL STATE}

In this section we consider the $\mathrm{Z}$ decays containing one or more $\gamma$ 's or a W boson (see table 2). They typically have branching ratios of order $10^{-10}\left(10^{-7}\right.$ for $\left.\mathrm{Z} \rightarrow \mathrm{XW}\right)$ in the Standard $\mathrm{Model}^{8}$, or are forbidden, as is the case for $\mathrm{Z} \rightarrow \gamma \gamma$. With such small branching ratios these decays should be far beyond the reach of
Table 1. Upper Limit on Branching Ratios at $95 \% \mathrm{CL}$ in Units of $10^{-5}$

\begin{tabular}{|l|r|r|r|}
\hline \multicolumn{1}{|c|}{ Decay } & $\mathrm{Z} \rightarrow \mathrm{e} \mu$ & $\mathrm{Z} \rightarrow \mathrm{e} \tau$ & $\mathrm{Z} \rightarrow \mu \tau$ \\
\hline Exp. & & & \\
\hline ALEPH & 1.0 & 8.0 & 5.5 \\
\hline DELPHI & 1.9 & 7.0 & 8.0 \\
\hline L3 & 1.5 & 3.0 & 2.9 \\
\hline OPAL & 4.6 & 7.2 & 35.0 \\
\hline \hline $\begin{array}{l}\text { Low Energy } \\
\text { Data }\end{array}$ & $\begin{array}{r}6.10^{-8} \\
\mu \rightarrow \text { eee }\end{array}$ & 10.0 & 5.0 \\
$\tau \rightarrow$ eee & $\tau \rightarrow \mu \pi$ \\
\hline
\end{tabular}

LEP; their detection would signal physics beyond the standard model. The limits for the

Table 2. Upper Limit on Branching Ratios at $95 \% \mathrm{CL}$ in Units of $10^{-5}$

\begin{tabular}{|c|c|c|c|c|}
\hline $\begin{array}{l}\text { Exp. } \\
\mathrm{Z} \rightarrow\end{array}$ & $\begin{array}{c}\text { ALEPH } \\
89-91\end{array}$ & $\begin{array}{c}\text { DELPHI } \\
90-91\end{array}$ & $\begin{array}{c}\text { L3 } \\
90-91\end{array}$ & $\begin{array}{l}\text { OPAL } \\
89-90\end{array}$ \\
\hline$\gamma \gamma$ & - & - & \multirow{2}{*}{12} & $\overline{14}$ \\
\hline$\pi^{0} \gamma$ & 16 & 15 & & 14 \\
\hline$\eta \gamma$ & 2.9 & 28 & 4.7 & 20 \\
\hline$\eta^{\prime} \gamma$ & 2.5 & - & 2.5 & - \\
\hline $\mathrm{J} / \psi \gamma$ & - & - & 2.9 & - \\
\hline$\gamma \gamma \gamma$ & 0.9 & 2.3 & $3.3^{f}$ & 6.6 \\
\hline$\pi W$ & 2.2 & - & $2.6^{*}$ & - \\
\hline $\mathrm{KW}$ & 2.2 & - & $2.6^{*}$ & - \\
\hline$\rho W$ & 2.6 & - & $4.1^{*}$ & - \\
\hline DW & 4.4 & - & - & - \\
\hline XW & 3.7 & - & - & - \\
\hline
\end{tabular}

branching ratios of $\mathrm{Z} \rightarrow \gamma \gamma, \pi^{0} \gamma, \eta \gamma$ and $\gamma \gamma \gamma$, with $\eta$ decaying into neutrals are obtained by observing deviations from the QED cross section for $e^{+} e^{-} \rightarrow \gamma \gamma(\gamma) \cdot{ }^{1,9-13}$ For L3, only the limit from the charged decay modes of $\eta$ is quoted. In the ALEPH analysis both neutral and charged decay modes of $\eta$ were studied, 
where $\eta \rightarrow \gamma \gamma$ or $3 \pi^{0}$ decays could be distinguished by their relatively broader electromagnetic showers in the detector compared to a $\gamma$ or a $\pi^{0}$. For $J$ and $W$ only the leptonic final states are considered since the hadronic decay modes suffer from a large $\mathrm{Z} \rightarrow q \bar{q}$ background. The measured limits on the branching ratios are about $10^{-5}$.

\section{SEARCH FOR NARROW RESONANCES}

The $l^{+} l^{-} \gamma$ and $q \bar{q} \gamma$ final states have been studied at LEP with no report of any anomalous yield for hard photons. The results have been interpreted in terms of the production of a narrow resonance $Y$ which decays into a pair of charged leptons or quarks. ${ }^{14-17}$ The upper limits on the product of the branching ratios $\mathrm{BR}(\mathrm{Z} \rightarrow \mathrm{Y} \gamma) \times \mathrm{BR}\left(\mathrm{Y} \rightarrow l^{+} l^{-}\right.$or $\left.q \bar{q}\right)$ are shown in Table 3. The L3 collaboration has also searched for final states with hadrons plus a scalar particle $\mathrm{S}$ that decays into two photons. To determine the limits on the branching ratio for this process a model is needed for acceptance calculations. One candidate is the Higgs boson which couples to two photons via loop diagrams involving charged fermions and $\mathrm{W}$ bosons. For this case an upper limit on the branching ratio of $3 \times 10^{-5}$ is obtained at the $95 \%$ confidence level.

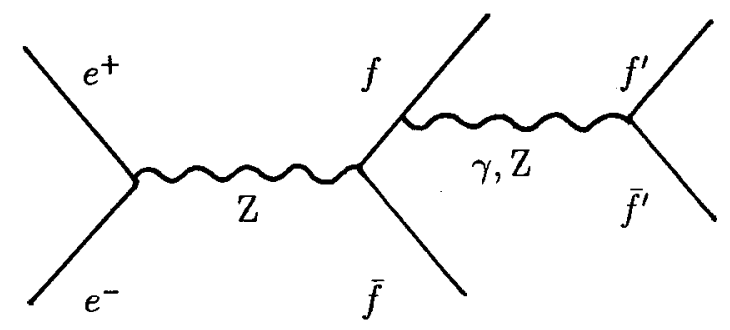

Figure 2. An example diagram for $e^{+} e^{-} \rightarrow f \bar{f} f^{\prime} \bar{f}^{\prime}$

\section{FOUR FERMION FINAL STATES- UPDATE ON ' $l \mathrm{~V}$ ' EVENTS}

An example Feynman diagram for four fermion final states is shown in figure 2. The cross section is dominated by diagrams where $f^{\prime} \bar{f}^{\prime}$ is produced by a virtual photon; hence in general it has a low invariant mass. Here we consider only the cases where $f \bar{f}$ is a pair of charged leptons and $f^{\prime} \bar{f}^{\prime}$ is a pair of oppositely charged particles of the same type. An example of the so called $l l \mathrm{~V}$ event is shown in figure 3 . In the initial study using their $89 / 90$

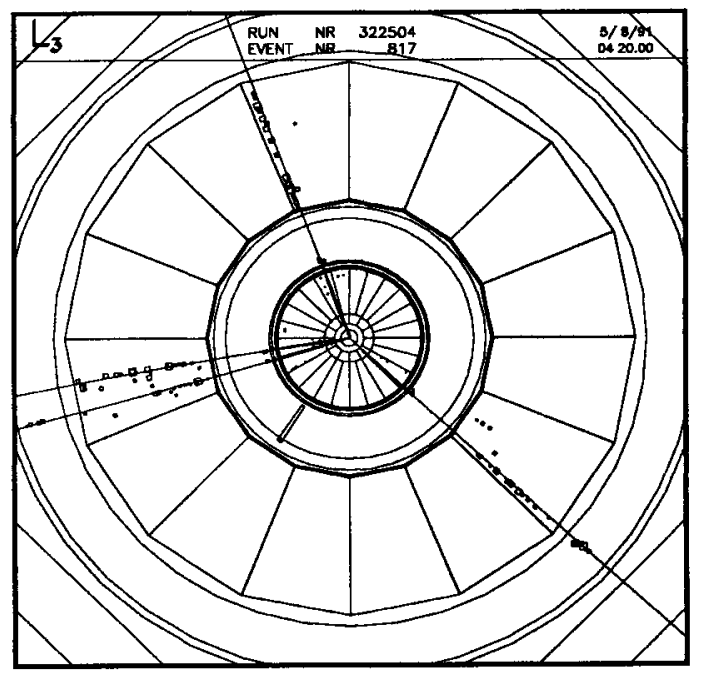

Figure 3. A $\mu^{+} \mu^{-} \mu^{+} \mu^{-}$event. Muon energies for wide angle pair are 35.1 and $40.6 \mathrm{GeV}$ and for the small angle ' $\mathrm{V}$ ' they are 9.0 and $7.2 \mathrm{GeV}$. The invariant mass of the ' $\mathrm{V}$ ' is about $2 \mathrm{GeV}$.

data ALEPH observed a significant excess of events in the $\tau \tau \mathrm{V}$ channel. ${ }^{18}$ Since then all four groups have analysed their data in this respect and the results are shown in table $4 .^{19,20}$ The ALEPH study using a larger sample still shows some excess in the $\tau \tau \mathrm{V}$ channel but with somewhat reduced significance. DELPHI data with lower statistics also exhibit excess in this channel. Based on the total numbers reported by the four LEP experiments some excess in the $\tau \tau \mathrm{V}$ channel is present but more data are 
Table 3. Upper Limit on Product of Branching Ratios at 95\% CL in Units of $10^{-5}$

\begin{tabular}{|l|c|c|c|c|c|c|}
\cline { 2 - 7 } \multicolumn{1}{c|}{} & $\mathrm{Y} \rightarrow \mathrm{e}^{+} \mathrm{e}^{-}$ & $\mathrm{Y} \rightarrow \mu^{+} \mu^{-}$ & $\mathrm{Y} \rightarrow \tau^{+} \tau^{-}$ & $\mathrm{Y} \rightarrow \mathrm{q} \overline{\mathrm{q}}$ & $\mathrm{H}^{0} \rightarrow \gamma \gamma$ & Mass range (GeV) \\
\hline DELPHI & - & - & - & 37 & - & $10-80$ \\
\hline L3 & 6.6 & 6.9 & 5.9 & 32 & 3.6 & $30-86$ \\
\hline OPAL & 11 & 7.1 & 11 & - & - & $65-83$ \\
\hline
\end{tabular}

Table 4. Observed and Expected number of $l l \mathrm{~V}$ Events

\begin{tabular}{|lc|rr|rr|rr|}
\hline & & \multicolumn{2}{|c|}{$e^{+} e^{-V}$} & \multicolumn{2}{|c|}{$\mu^{+} \mu^{-} \mathrm{V}$} & \multicolumn{2}{|c|}{$\tau^{+} \tau^{-} \mathrm{V}$} \\
Experiment & Period & data & MC & data & MC & data & MC \\
\hline \hline ALEPH & $(1989-1991)$ & 18 & 18.2 & 18 & 15.1 & 18 & 7.4 \\
\hline DELPHI & $(1990-1991)$ & 11 & 9.9 & 9 & 9.3 & 8 & 3.9 \\
\hline L3 & $(1991)$ & 8 & 6.3 & 5 & 4.0 & 4 & 4.0 \\
\hline OPAL & $(1990-1991)$ & 21 & 27.7 & 18 & 15.3 & 11 & 9.5 \\
\hline \hline \multicolumn{2}{|c|}{ T T O T A L } & 58 & 62.1 & 50 & 43.7 & 41 & 24.8 \\
\hline
\end{tabular}

needed to shed light on this question.

\section{ADDITIONAL HEAVY NEUTRAL GAUGE BOSON}

Finally the L3 results on the heavy gauge boson $Z^{\prime}$ are presented. Most extensions to the Standard Model involve such a gauge boson. In general the $\mathrm{Z}$ mass and its couplings to the fermions are affected through $\mathrm{ZZ}^{\prime}$ mixing. One can obtain indirect information on the $Z^{\prime}$ parameters by performing a model independent fit to the data and comparing it with the Standard Model. A more direct approach, adopted in the L3 analysis, is to modify the relevant formulae for cross sections and asymmetries to incorporate the $\mathrm{Z}^{\prime}$ and then fit to the data. ${ }^{21}$ The additional parameters involved are the $Z^{\prime}$ mass $M_{Z^{\prime}}$, the $Z Z^{\prime}$ mixing angle $\Theta_{M}$ and a model dependent parameter related to the $\mathrm{Z}^{\prime}$ couplings to the fermions. Two well known models are the $\mathrm{E}_{6}$ :

$\underbrace{\mathrm{SU}(3)_{\mathrm{c}} \otimes \mathrm{SU}(2)_{\mathrm{L}} \otimes \mathrm{U}(1)_{\mathrm{Y}}}_{\text {StandardModel }} \otimes \underbrace{U(1)_{\chi} \otimes U(1)_{\psi}}_{Z^{\prime}=\cos \Theta_{6} \chi+\sin \Theta_{6} \psi}$ where $\Theta_{6}$ is the model parameter and the Left-
Right Model: $\mathrm{SU}(2)_{\mathrm{L}} \otimes \mathrm{SU}(2)_{\mathrm{R}} \otimes \mathrm{U}(1)_{\mathrm{B}-\mathrm{L}}$ with the parameter $\alpha_{\mathrm{LR}}$.

The 90/91 data of L3 were analyzed for all possible values of

$-\pi / 2 \leq \Theta_{6} \leq \pi / 2$ and

$\sqrt{ } 2 / 3 \leq \alpha_{\text {LR }} \leq \sqrt{ } 2$

A top quark mass of $150 \mathrm{GeV}$, Higgs mass of $300 \mathrm{GeV}$ and an $\alpha_{\mathrm{s}}$ value of 0.12 were used in the study. Figure 4 shows the excluded values of $\Theta_{\text {mix }}$ and $\Theta_{6}$ for the $E_{6}$ model for the values of $\mathrm{M}_{\mathrm{Z}^{\prime}}$ greater than 200 and $700 \mathrm{GeV}$. For $\Theta_{6}=0$ ( $\chi$ model $)$ the excluded values of $\Theta_{M}$ are shown in figure 5 for a given $\mathrm{M}_{\mathrm{Z}^{\prime}}$. From this plot $\mathrm{M}_{\mathrm{Z}^{\prime}}$ must be greater than $120 \mathrm{GeV}$. The excluded values of $\Theta_{M}$ and $\alpha_{L R}$ are given in figure 6.

\section{CONCLUSIONS}

Vast amount of data have been analyzed by all four LEP experiments in the last three years. No new particles or anomalously high branching ratios for rare decays of the $\mathrm{Z}$ have been reported. Upper limits at $95 \%$ CL of order $10^{-5}$ have been set on the branching ratios 


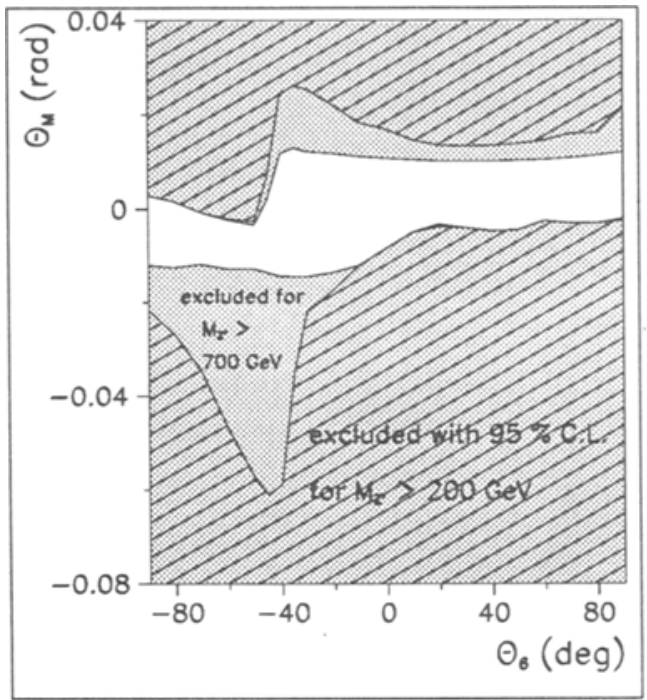

Figure 4. Excluded values of $\Theta_{\text {mix }}$ and $\Theta_{6}$ for $M_{Z^{\prime}}>$ $200 \mathrm{GeV}$ (crossed region) and for $\mathrm{M}_{\mathrm{Z}^{\prime}}>700 \mathrm{GeV}$ (dotted region) for the $\mathrm{E}_{6}$ model.

for lepton flavour violating $\mathrm{Z}$ decays, rare $\mathrm{Z}$ decays, and the production of new narrow resonances in the mass range $10-86 \mathrm{GeV}$. Some excess in the $\dot{\tau} \tau \mathrm{V}$ topology is still present and awaits more LEP data for clarification. The $\mathrm{ZZ}^{\prime}$ mixing angle is limited to $\pm 1.5^{\circ}$ for most models.

\section{ACKNOWLEDGMENTS}

I am indebted to many colleagues who have helped me in preparing this talk, especially T.E. Coan, M. Green, H. Jannsen, J. Qian, F. Richard, S. Riemann, O. Rind, A. Rubbia, S. Shevchenko, V. Shoutko and S.L. Wu for providing me with preliminary data. I am grateful to Dr R. Kleiss for a helpful discussion. I would like to acknowledge the support of the National Science Foundation for this work.

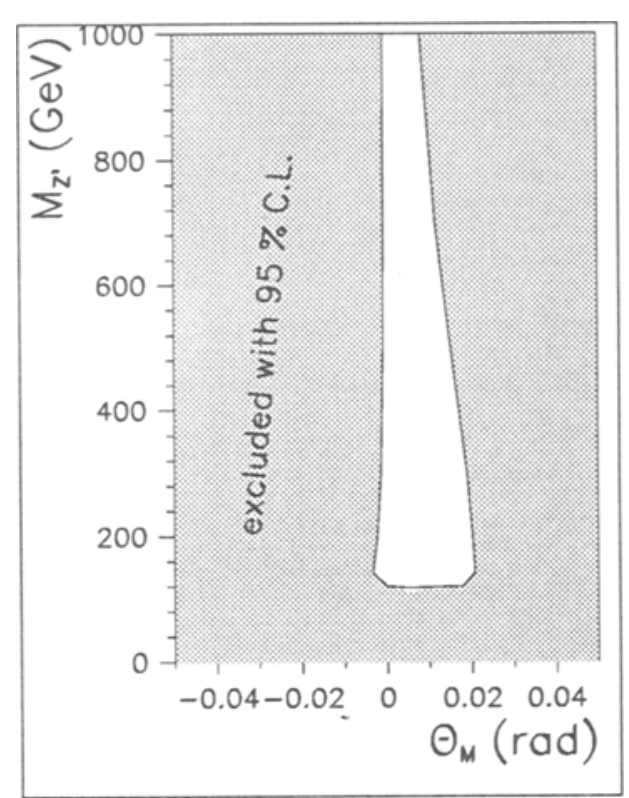

Figure 5- Excluded values of the $M_{Z^{\prime}}$ and $\Theta_{M}$ for the $\chi$ model.

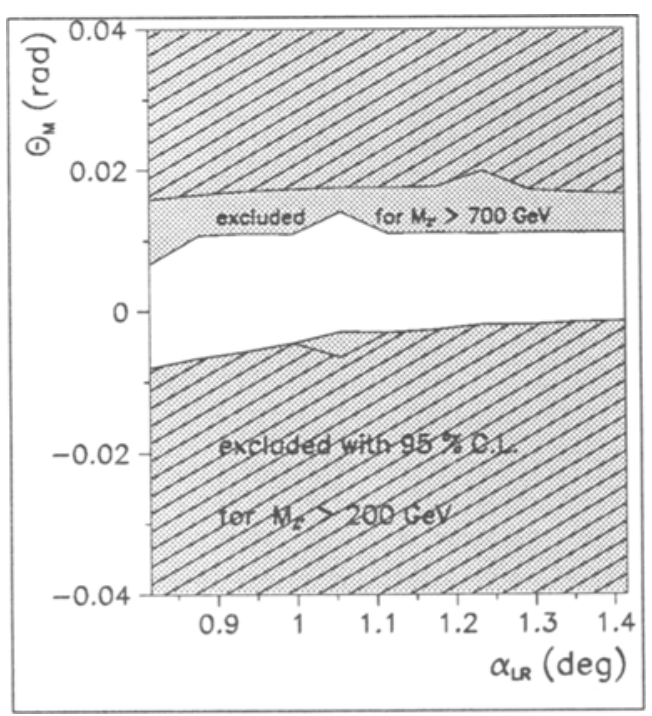

Figure 6. Excluded values of $\Theta_{M}$ and $\alpha_{L R}$ for $M_{Z^{\prime}}>$ $200 \mathrm{GeV}$ (crossed region) and for $\mathrm{M}_{\mathrm{Z}^{\prime}}>700 \mathrm{GeV}$ (dotted region) for the Left-Right model. 


\section{REFERENCES}

1. T. K. Kuo and N. Nakagawa, Phys. Rev. D32 (1985) 306;

G. Eilam and T. G. Rizzo, Phys. Lett. B188 (1987) 91.

2. ALEPH Collab., Search for rare decays of the $\mathrm{Z}$ boson, Present conference.

3. DELPHI Collab., A search for lepton flavour violation in $\mathrm{Z}^{0}$ decays, Present conference.

4. OPAL Collab., M.Z. Akrawy et al. Phys. Lett. B 254 (1991) 293.

5. L3 Collab., B. Adeva et al., Phys. Lett. B 271 (1991) 453; updated results.

6. E.W.N. Glover, J.J. van der Bij, Rare $\mathrm{Z}$ decays, $\mathrm{Z}$ Physics at LEP1, ed. G. Altarelli, R. Kleiss and C. Verzegnassi, CERN 89-08, Vol. 2 (1989) 1.

7. Particle Data Group, Phys. Rev. D 45 (1992).

8. L. Arnellos et al., Nucl. Phys. B 196 (1982) 378 ;

A. Manohar, Phys. Lett. B244 (1990) 101;

W. Marciano And D. Wyler, Z. Phys. C3 (1979) 181.

9. L3 Collab., O. Adriani et.al, Phys. Lett. B288 (1992) 404.
10. ALEPH Collab. Search for excited quarks and the decays $\mathrm{Z} \rightarrow \gamma \gamma \gamma$ and $\mathrm{Z} \rightarrow \operatorname{gg} \gamma$, Present conference.

11. OPAL Collab.,M.Z. Akrawy et.al., Phys. Lett. B257 (1991) 531.

12. DELPHI Collab., P. Abreu et. al. ,Phys. Lett. B268 (1991) 296.

13. DELPHI Collab., Search for new phenomena with photon channels from $\mathrm{Z}^{0}$ decays, Present conference.

14. L3 Collab., B. Adeva et al., Phys. Lett. B262 (1991) 155.

15. L3 Collab., O. Adriani et al., CERN preprint CERN-PPE/92-131.

16. DELPHI Collab., P. Abreu et al., Z. Phys. C53 (1991) 555.

17. OPAL Collab., P.D. Acton etal., Phys. Lett. B273 (1991) 338.

18. ALEPH Collab., D. Decamp et al., Phys. Lett. B263 (1991) 112.

19. OPAL Collab., P.D. Acton etal., Phys. Lett. B287 (1992) 369.

20. DELPHI Collab., A search for $Z^{0} \rightarrow$ $l^{+} l^{-} \mathrm{V}$, Present conference.

21. A. Leike, S. Riemann, T. Riemann, CERN preprint CERN-TH 6545/92. 